\title{
ROBOTS! - Freshman Clinic in Mechanical Engineering
}

\author{
Hong Zhang Jennifer Kadlowec \\ College of Engineering, Rowan University, Glassboro, NJ 08028 \\ zhang, kadlowec@rowan.edu
}

\section{Introduction}

The integration of both hands-on experience and minds-on thinking in engineering education is the hallmark of Rowan Engineering. The Engineering Clinic ${ }^{1,2}$ sequence was developed to promote project-based learning. As a unique component of the Rowan engineering curriculum, engineering clinic is a framework that includes the topics from introduction of engineering at different levels to junior/senior design projects usually seen in a normal engineering program. The details of the clinics will vary for different disciplines and different academic years, but all of them will be based on hands-on education.

Modeled under medical clinic rotation, every Rowan engineering student needs to attend one clinic project each semester throughout his/her four-year undergraduate study. The topics of the clinics can vary broadly. In the first year, through simple hands-on projects such as reverse-engineering a hand-powered radio, brewing beer $^{3}$, or building a small Soccerbot, the freshmen will understand each field of engineering better and develop stronger interest in engineering overall. In sophomore clinic, students will be asked to work in multidisciplinary groups and build a pre-designed system such as a yeast-based fuel-cell-powered Lego robot. For juniors and seniors, because they already have the basic theoretical background, they will be teamed for real life engineering projects. These projects can be either based on the research conducted by faculty members or obtained from industrial sponsors. Under the direction of a principal manager (a faculty member), each team needs to finish the project within certain timeline, generally a semester or a year.

In this paper, we will illustrate how we took advantage of robot to introduce Mechanical Engineering to the freshmen with the form of engineering clinic.

After this introduction, the second section of this paper begins provides an detailed explanation of the idea and management of freshman clinic. In the third section, we will provide more detail of using robot as a tool to introduce Mechanical Engineering. In the fourth and last section, we will highlight some impacts and benefits of the project.

\section{Freshman Clinic}

Generally speaking, the freshman clinic corresponds to the Introduction to Engineering in many other universities, though in a unique format. It consists of two parts. The first one is the teaching of basic engineering skills and ethics that will be essential to students' success or even survival in engineering school and their future 
engineering career. The topics include review of high school mathematics and unit conversions, use of the spreadsheet like Microsoft Excel to analyze data and draw figures, and practice of writing reports and making presentations. The format for covering these topics fits into a one-hour lecture at each Monday for the whole semester.

The other part of the clinic is to introduce different disciplines of engineering to the freshmen. There are four departments in Rowan Engineering: Mechanical Engineering (ME), Electrical and Computer Engineering (ECE), Civil Engineering (CE), and Chemical Engineering (ChE). Although most students had already made their minds of their career directions and declared their majors before registration, many were still struggling to decide which engineering majors suited their interest and career goal better. Meanwhile, many freshmen chose their majors just by visiting the university open house or consulting their parents and friends without truly understanding the opportunities and responsibilities of being an engineer at their choice. This clinic will help them to obtain more information and evaluate their choices if they could have a chance to take a closer look at each engineering discipline themselves. Even for those who were well informed and firmly determined, it will still be a great opportunity to personally experience the other departments. Although even laypersons will have some vague idea associated with each subject of the engineering, it is often beyond their imagination to realize what each major is really doing. For example, when we asked the visiting high school students in the university open house about what was their impression of mechanical engineering, a common answer was to repair their cars. Indeed, few of them could recognize that robotics or even thermal and fluids were within the realm of Mech anical Engineering. Further, with the development of the technology, most projects today require cooperation from many disciplines. It will be advantageous to the students to have a broad knowledge beyond their own majors.

The arrangement of the freshman clinic is somewhat different from the traditional approach of the engineering education. It is organized after the clinic internship of hospitals. That is, each engineering department prepares a three-week module that highlights their respective discipline. During the semester, the module will be given simultaneously and repetitively, while the students rotate through these modules. At the end, they will have a chance to experience all the four majors of engineering that are offered in Rowan.

On the other hand, as we mentioned earlier, it is the tradition of Rowan Engineering to emphasize the hands-on experience throughout the curriculum. Therefore, it is the consensus of the four departments to provide experiments or other hands-on experiences in all modules. After several years' iterations, the module of Mechanical Engineering is settled to current configuration, i.e., the projects based on robots: a manually controlled Soccer-bot (Figure 1) and a microprocessor based BOEBot (Figure 2).

The Soccerbot is a miniature legged-robot with a handheld controller. Two DC motors control two symmetric sets of moving components, left and right, respectively. Each set includes two pairs of gears to obtain desired speed, two levers to achieve proper gaits, and three legs to support the body and complete the motion. Via a tether, the two sets of the legs can be controlled separately using two sticks of the control box. Strictly speaking, the Soccerbot is not a real robot because of the lack of intelligence. However, it is still a great system to demonstrate many engineering theories. 


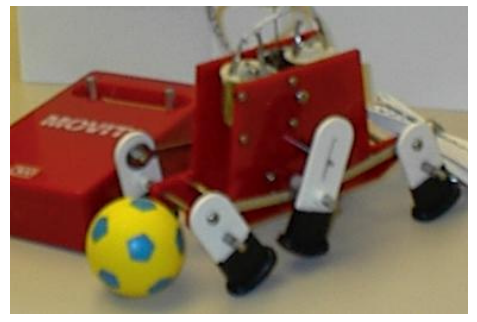

Figure 1: Soccerbot

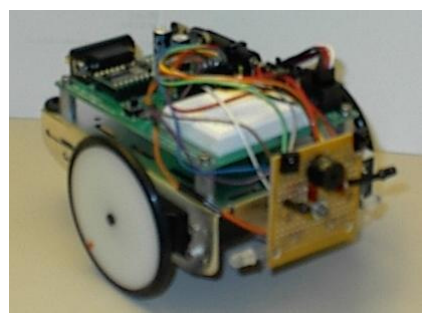

Figure 2: BOEBot

The BOEBot, or Board Of Education robot is a more advanced system compared to the Soccerbot. A typical BOEBot includes three parts: a chassis, a computer board, and an additional electronic board. The aluminum chassis supports two driving wheels that are independently controlled by two modified servos and one small balancing roller. On top of the chassis is a computer board based on a small chip called Basic Stamp II, a microprocessor from Parallax Inc. It comes with 2K EPROM and 32byte RAM, and can run at the speed of $20 \mathrm{MHz}$ or 4000 instructions per second. The programming language of Basic Stamps is PBASIC, a simplified version of BASIC. A user could develop and debug programs in the integrated programming environment in a $\mathrm{PC}$, which is connected to the Basic Stamp via serial ports on both sides. The PC will compile the program, translate it to executable code, and then download the code to the EEPROM of the Stamp. Besides the serial port, the Basic Stamp II has 16 Input/Output pins that can communicate with the external devices independently. The electronic board of the BOEBot is the carrier of the most on-board equipment. In our setting for the freshman clinic, we put two bicolor LED lights, a speaker, two photo-resistors, and two pairs of infrared sensors. For advanced applications, more sophisticated sensors such as sonar and camera can also be used to provide richer information.

\section{Using Robot in Mechanical Module}

With robots as the core of our module, we divided the freshman clinic to four 30 minutes long mini lectures and three 150 minutes units of hands-on experiences. The topics of the lectures included introduction to the robot, simple mechanisms, material selection for common engineering purpose, and sensors. The hands-on exercises were assembling the Soccerbot, applying Solidworks, a kind of popular CAD software to draw the control box of the Soccerbot, and programming and controlling the BOEBot. During each week of the module, we would provide one to two mini lectures and one major unit to the students.

In the first week, we gave students a brief introduction of robots. Since the students just left high school merely four months ago, we kept the introduction simple but as informative as possible. Rather than giving technical details, we started from sci-fi characters like R2D2 and Terminator to intrigue their interests, and drew analogies between robot and human being to help them understand the ideas.

After this discussion, we then provided them basic mechanical background of gears with the transmission of the Soccerbot as the major example. For the mechanical 
students, they would learn more details about the gears in their future study. However, it might be the only chance for the other students to clarify their ideas of gear ratio of transmissions and to see different types of gears.

After two mini lectures, the students would begin to assemble the Soccerbot with the kits provided. According to the management of the freshman clinic, every student was asked to answer a carefully designed questionnaire at the beginning of the semester. According to their answers, we could determine their learning attitudes and group them accordingly with the help from the Center for Advancement of Learning at Rowan University. In each group, there should be at least one student who was strong in handson activity and one willing to take lead even facing unknown area. Throughout the semester, the students were required to stay in the same group and work with the same team members. Therefore, they would know each other well, and not need to spend the precious time to build new teams again and again.

When the students started to construct the Soccerbot, they were required to read the instruction, figure a plan, and distribute the work evenly within the group. Meanwhile, they would measure the size and count the numbers of the teeth of the gears to be used, calculate the gear ratios between motors and axles of legs, and draw an illustration of the power train. In a typical four-person group, two students would work on the left and right parts of the body, which is symmetric, another one would build the control box, and the fourth would finish the drawing of the gear train. When the robots were finished, the students should conduct several experiments such as determining the maximum speed of the robot, and more interestingly, playing a robot soccer game at the end of the session.

During the second week, we introduced the students to mechanical design, which included a mini lecture of material selection and a major project of engineering drawing. With the example of the Soccerbot, we discussed various types of materials, their properties and functions, and the way to choose them for different purposes. After that, we would bring them to the computer lab for a short course of engineering drawing using Solidworks. The students were first directed through a tutorial of the software, and then they would try themselves to draw the Soccerbot control box, a shelled box with various extrusions and openings.

Finally, we would move to the more sophisticated BOEBot in the third week. Before playing with it, we gave a brief presentation about sensors and their applications on robots. Then we started to teach them simple programming skills to control the robot. Since the time was short, we would like the students to grasp the concept of programming instead of venturing through the detail of each command. Further, as engineers instead of computer programmers, our students should focus on a firm understanding of the interaction between software and hardware. Therefore, we provided them a lookup table of the commands. (See Table 1.) With this table, they just needed to understand what they wanted the robot to do and outline the program in blocks or flow charts. Then they could search the table, fill in the real command, and write the final code.

\begin{tabular}{|l|l|}
\hline \multicolumn{1}{|c|}{ Command Block Description } & \multicolumn{1}{|c|}{ Contents of Block } \\
\hline FORWARD - Move forward for about 4mm. & PULSOUT 12, 500 \\
& PULSOUT 13, 1000 \\
PAUSE 20
\end{tabular}

Proceedings of the 2002 American Society for Engineering Education Annual Conference \& Exposition Copyright (c) 2002, American Society for Engineering Education 


\begin{tabular}{|l|l|}
\hline TURN RIGHT - spin clockwise for about $3^{\circ}$ & $\begin{array}{l}\text { PULSOUT 12, 1000 } \\
\text { PULSOUT 13, 1000 } \\
\text { PAUSE 20 }\end{array}$ \\
\hline BEEP SPEAKER -Beep the speaker for tms at frequency freq & FREQOUT 11, t, freq \\
\hline $\begin{array}{l}\text { Measure light level from left photo-resistor, and record the output } \\
\text { to variable left_photo with word size }\end{array}$ & $\begin{array}{l}\text { HIGH 5 } \\
\text { PAUSE 5 } \\
\text { RCTIME 5, 1, left_photo }\end{array}$ \\
\hline$\ldots .$. & $\ldots$ \\
\hline
\end{tabular}

Table 1: A portion of the lookup table of correspondences between hardware actions and software commands

After a quick explanation of the hardware, the controlling method, and their corresponding codes, we introduced several syntax related commands such as the loop and flow control. After presenting each new command, we would give students a small project to apply to the robot. Within two hours, the students could direct the robot to move forward and backward, turn around, flash lights, and sing a song. Moreover, after applying the newly learned knowledge of sensor such as a photo-resistor, they could also make the robot to automatically circumvent an obstacle such as a hand.

\section{Impact and benefit}

Due to the background requirement, we can navigate more advanced theories and experiments progressively only throughout the four years of college. Hence, more intuitive knowledge and straightforward methods have to be used for the first semester of the first year in the students' voyage of higher education. Therefore, selecting the most suitable project in the freshman clinic is the key to success. More traditional approaches often restrict themselves to simple and pure mechanical systems such as analyzing and assembling a gearbox. However, there are some major drawbacks of this approach. First, students may not fully understand the purpose or fully recognize the importance of the project, hence may show little if any interest. Second, for the students who have already declared or will declare the non-ME majors, they may unwillingly limit their understanding of mechanical engineering based on the freshman clinic. The oversimplified approach tends to bring these students certain bias toward the discipline that they will not encounter in future.

Our idea is to put the projects of the mechanical engineering in a self-contained, sophisticated, yet fundamental framework. It should be interesting enough to inspire students, broad enough to expose them the full spectrum of Mechanical Engineering, and still simple enough to help them focus and finish within three three-hour modules. Our final selection, after some iterations and external references ${ }^{4}$, is to use robot as the platform. As a common topic of many sci-fi movies or stories, robots carry a strong hallmark of science and technology. As we found in the clinic, the students showed great interest toward the subject. Meanwhile, different from regular scientific experiments whose goals are to measure predefined quantities or to verify given properties, our project is to assemble or program a robot and then control its movement. The result of their work will be visible not only by themselves and the instructors, but also by the whole class. Every group would show great pride after they successfully finished assembling the 
Soccerbot or running the BOEBot. Further, the projects on robot also show many freshmen a different image of the mechanical engineering from what they had in mind. They will also understand that on the contrary to stereotypes, modern engineering is an integrated system with all the different majors interlaced and interacting together. They will see that even a toy-like small robot is a combination of electro-mechanics, materials, and computer programming. They will also learn that Mechanical Engineering could be pragmatic, exciting and fun.

\section{References:}

1. K. Jahan, R.A. Desseau, R.P. Hesketh, A, J. Marchese, R.P. Ramachandran, S.A Mandayam and J.L. Schmalzel, "Engineering Measurements in the Freshman Engineering Clinic at Rowan University", ASEE Annual Conference and Exposition, Seattle, Washington, Session 1326, June 28 - July 1, 1998.

2. A. J. Marchese, J. A. Newell, R. P. Ramachandran, B. Sukumaran, J.L. Schmalzel and J. Marriappan, "The Sophomore Engineering Clinic: An Introduction to the Design Process Through a Series of Open Ended Projects", ASEE Annual Conference and Exhibition, Charlotte, North Carolina, Session 2225, June 20 - 23, 1999.

3. S. Farrell, R. P. Hesketh, J. A. Newell, and C. S. Slater, Introducing Freshmen to Reverse Process Engineering and Design through Investigation of the Brewing Process", International Journal of Engineering Education. 17(6), 2001.

4. L. Hrebien, R. Carr, Integrating the Engineering Curriculum through a Design Contest, ASEE Mid-Atlantic Region Spring Meeting, Glassboro, New Jersey, April 21, 2001 